\title{
Class-specific responses of brown adipose tissue to steroidal and nonsteroidal mineralocorticoid receptor antagonists
}

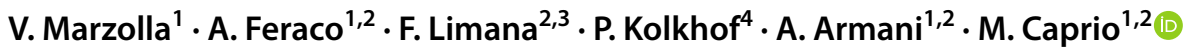

Received: 9 May 2021 / Accepted: 7 July 2021 / Published online: 16 July 2021

(C) Italian Society of Endocrinology (SIE) 2021

\begin{abstract}
Aldosterone exerts deleterious effects on the cardiovascular system and promotes adipose tissue expansion via mineralocorticoid receptor (MR) activation. We previously demonstrated that administration of steroidal mineralocorticoid receptor antagonists (MRA) in mice fed a moderate high-fat diet is able to reduce white adipose tissue (WAT) expansion, stimulate browning of WAT and activate interscapular brown adipose tissue (iBAT). Here, we aimed to compare the metabolic and adipose tissue-specific effects of the novel non-steroidal MRA finerenone (Fine) and spironolactone (Spiro) in a mouse model of very high-fat diet (HFD)-induced obesity. C57BL/6 J male mice were fed a 60\% HFD containing or not Spiro or Fine for 12 weeks. WAT and iBAT morphology and adipose tissue gene expression analysis were assessed. After 12 weeks, both groups of mice showed similar weight gain compared to the HFD group. Histological and molecular analyses of WAT did not show significant differences among all experimental groups; differently, iBAT histological analysis revealed that Fine was able to increase recruitment of brown adipocytes in this depot, whereas mice treated with Spiro failed to elicit any iBAT response, as indicated by no changes in lipid droplets size and iBAT density, compared to HFD. Increased iBAT recruitment could explain, at least in part, the improved insulin resistance observed in mice treated with Fine, as shown by a significant reduction in homeostasis model assessment of insulin resistance (HOMA) index. These findings were confirmed by gene expression analysis of $u c p-1, p g c 1-\alpha$, and beta-3 adrenoreceptor (Adrb3) in iBAT, revealing significantly higher expression of these thermogenic genes in HFD + Fine group compared to HFD, whereas Spiro did not modify their expression. In summary, we demonstrated that, differently from Fine, Spiro did not induce iBAT recruitment. Our current study suggests that Fine, through its direct effects on iBAT, represents a promising pharmacologic tool to treat human metabolic diseases associated with adipose tissue dysfunctions.
\end{abstract}

Keywords Aldosterone $\cdot$ Obesity $\cdot$ Adipocyte $\cdot$ Mineralocorticoid receptor $\cdot$ Cardiometabolic rehabilitation

M. Caprio

massimiliano.caprio@sanraffaele.it

1 Laboratory of Cardiovascular Endocrinology, San Raffaele Research Institute, IRCCS San Raffaele Roma, Via di Val Cannuta, 247, 00166 Rome, Italy

2 Department of Human Sciences and Promotion of the Quality of Life, San Raffaele Roma Open University, Rome, Italy

3 Laboratory of Cellular and Molecular Pathology, IRCCS San Raffaele Roma, Rome, Italy

4 Bayer AG, R\&D Preclinical Research Cardiovascular, Wuppertal, Germany

\section{Introduction}

Obesity and its related comorbidities have become major public health concerns, particularly during the COVID-19 pandemic. Aldosterone (Aldo), besides its well-known renal effects, exerts deleterious actions on the cardiovascular system and increases cardiovascular risk [1]. Likewise, elevated circulating Aldo levels correlate with obesity, suggesting a causal relationship between increasing body weight and Aldo production [1]. Aldo acts through mineralocorticoid receptor (MR), a cytosolic transcription factor which, upon activation, translocates to the nucleus to activate transcription of specific genes. It has been well established a key role for MR in white adipose tissue (WAT) and brown adipose tissue (BAT) functions [1]. Indeed, we and others demonstrated that classical steroidal MR antagonists (MRAs) 
administration in mice fed a moderately high fat diet (42\% $\mathrm{kcal}$ from fat) was able to reduce WAT expansion, induce interscapular BAT (BAT) activation and stimulate browning of WAT [2]. Importantly, we recently showed that treatment of a mouse model of very high-fat diet-induced obesity (HFD, 60\% kcal from fat) with the novel non-steroidal MRA finerenone (Fine) led to increased multilocularity and expression of thermogenic markers at the level of iBAT with concomitant improvement in glucose tolerance, without affecting WAT mass and white adipogenic marker gene expression, demonstrating that Fine induces BAT-specific effects [3]. The aim of the present study was to compare the metabolic and adipose tissue-specific effects of the classical steroidal MRA spironolactone (Spiro) versus the novel non-steroidal MRA Fine in a mouse model of HFD-induced obesity.

\section{Materials and methods}

\section{Animal model}

Animal procedures were approved by the Italian National Institutes of Health Care and Use Committees (approval number 493/2016-PR). Male 10-week old C57BL/6 J mice (Charles River Laboratories, Calco, Italy) were fed a very high-fat diet (HFD) $(60 \% \mathrm{kcal}$ as fat; D12492; Research Diets) or HFD (D12492) containing Spiro $(0,165 \mathrm{gr} / \mathrm{Kg}$ of diet) or Fine (100 ppm, i.e. $0.1 \mathrm{~g}$ of Fine $/ \mathrm{kg}$ of diet) for 12 weeks. Mice were divided into 3 groups $(n=10)$ as follows: mice fed a HFD (HFD group); mice fed a HFD plus Spiro (HFD group + Spiro group); mice fed a HFD plus Fine (HFD + Fine group).

\section{Gene expression analysis}

Total RNA was isolated from snap-frozen iBAT and WAT (inguinal and gonadal fat) depots using RNeasy lipid tissue mini kit (Qiagen, Milan, Italy) following the manufacturer's instructions. The purity, integrity and yield of RNA were analyzed by Agilent Technologies 2001bioanalyzer using the RNA 6000 LabChip kit. Total RNA $(1 \mu \mathrm{g})$ was treated with RNase-Free DNase 1 (Qiagen) and reverse transcribed using High-Capacity cDNA Reverse Transcription System (Applied Biosystems (Thermo Fisher Scientific), Milan, Italy) according to the manufacturer's instructions. Quantitative real-time-PCR (qRT-PCR) assays were performed in 96-well optical plates using Mx3000P LightCycler instrument (Stratagene, Milan, Italy). Each cDNA sample was analyzed in duplicates using gene-specific primers spanning intron/exon boundaries for gene expression quantification and Fast SYBR green Master Mix (Applied Biosystems (Thermo Fisher Scientific), Milan, Italy). Quantitative normalization of cDNA in each sample was performed using TATA-box binding protein as an internal control. Relative quantification was calculated using the $2^{-\Delta \Delta \mathrm{CT}}$ method.

\section{Histological and immunostaining analyses of fat depots}

Dissected iBAT and WAT depots were fixed overnight in $10 \%$ formaldehyde at $4{ }^{\circ} \mathrm{C}$ and rinsed with $0.1 \mathrm{M}$ phosphate buffer, $\mathrm{pH} 7.4$, before being embedded in paraffin. Each paraffin-embedded depot was cut ( $7 \mu \mathrm{m}$ thick) and the cutting plane corresponded to the largest surface used for histological examination and morphometry evaluated by hematoxylin-eosin (H\&E) staining. 10 fields at 20-fold magnification for each slide were analyzed to determine the mean adipocyte area (for WAT) and lipid droplet size (for iBAT) using a digital image system (Adiposoft - ImageJ, open-source software). iBAT density (the percentage of adipocyte area positive for eosin staining) was determined using ImagePro Premier software (Media Cybernetics). Immunohistochemistry of iBAT for uncoupling protein-1 (UCP-1) was carried out on tissue sections fixed as above described. Slides were processed on the Autostainer Link 48 (Agilent Technologies, Santa Clara, CA) and prepared using EnVision FLEX Target Retrieval Solution, Low pH (Dako, Glostrup, Denmark). Six fields at 20-folds magnification for each slide were analyzed to determine UCP-1 positive staining area using ImagePro Premier software (Media Cybernetics).

\section{Insulin resistance assessment and serum analysis}

At the time of kill, peripheral blood samples were collected via retroorbital bleeding. Fasting serum samples were assayed for total cholesterol, triglycerides, free fatty acid (data not shown), glucose and insulin by colorimetric assay (Plaisant Srl, Rome, Italy). The HOMA-IR (homeostasis model assessment of insulin resistance) index was calculated as [fasting serum glucose concentration $(\mathrm{mg} / 100 \mathrm{ml}) \times$ fasting serum insulin concentration (mIU/L) / 405] to assess insulin resistance [4].

\section{Statistical analysis}

Data are reported as means \pm standard error of the mean (SEM). Data points $> \pm 2$ standard deviation (SD) from the mean were considered statistical outliers and were excluded from all analyses (ROUT method). Statistical comparisons 
were performed by one-way ANOVA tests, followed by Bonferroni multiple comparison post hoc analysis, using Prism 8.0 (GraphPad, San Diego, CA, USA). Values of $P<0.05$ were considered significant.

\section{Results and discussion}

In the present study, wild-type mice were fed a HFD or HFD containing Spiro or Fine for 12 weeks. HFD + Spiro group, as well as HFD + Fine group, showed similar weight gain compared to HFD group (data not shown). Interestingly, we recently demonstrated that Fine exerts a direct effect on iBAT recruitment, reducing iBAT lipid droplets size and increasing BAT marker expression, in mice fed a HFD, as compared to HFD group [3]. To assess whether Spiro was able to induce comparable effects on iBAT in a murine model of HFD-induced obesity, we performed histological analysis and molecular characterization of iBAT depots in all groups of mice. Interestingly, iBAT histological examination of mice treated with Spiro showed a lack of iBAT response in mice fed a very HFD, as indicated by no changes in lipid droplet size (Fig. 1B) and iBAT density (Fig. 1C), compared to HFD. Differently, histological analyses showed that Fine was able to increase iBAT recruitment in mice fed a very HFD diet. Such increase could be responsible, at least in part, for the reduction in insulin resistance observed in mice treated with Fine, as shown by a significant reduction in HOMA-IR index compared to Spiro- and HFD-groups. (Fig. 1D). Such findings were further supported by qRT-PCR analyses of thermogenesis-related genes ucp-1, peroxisome proliferator-activated receptor gamma coactivator 1-alpha (pgcl- $\alpha$ ), beta-3 adrenoreceptor (Adrb3) and UCP-1 immunostaining in iBAT, revealing a significantly increased expression of thermogenic genes (Fig. 1E) and UCP-1immunostaining (Fig. 1G) in HFD + Fine group compared to HFD (Fig. 1D), whereas Spiro did not alter the expression of these genes (Fig. 1E), nor UCP-1 immunostaining (Fig. 1G). Accordingly, qRTPCR analysis showed reduced expression of white adipocyte-specific marker genes (leptin and adyc5) in iBAT of HFD + Fine group, as compared with HFD (Fig. 1F), with no differences between HFD + Spiro and HFD group, indicating that Fine, but not Spiro, is capable to enhance iBAT recruitment in our experimental model. To evaluate whether Spiro and Fine were able to induce browning of WAT, we performed histological analysis of inguinal and gonadal depots. Histological analyses revealed that both Spiro and Fine did not induce any morphological change in both inguinal and gonadal fat depots, (Fig. 2A and B). Indeed,
HFD + Spiro and HFD + Fine group did not show any difference in adipocyte size compared to HFD group (Fig. 2A and Fig. B). In accordance with these data, mRNA levels of white and brown adipocyte markers did not significantly differ among all experimental groups (Fig. 2C),

In summary, our study demonstrates that only treatment with Fine, not with Spiro, improves insulin resistance in a model of very HFD-induced obesity in mice. Differently from Fine, the classical MRA Spiro was not able to induce iBAT recruitment in this experimental model. Notably, a recent study showed that treatment of healthy non-obese subjects with Spiro promoted BAT activation after cold exposure, demonstrating that MRAs are able to activate BAT also in humans [5]. These data are in accordance with our previous study, where Spiro determined increased activity of iBAT in a mouse model fed a moderately high-fat diet [2], with a concomitant reduction in body weight gain and improvement of glucose tolerance. It is important to remark that in the present study, where mice were fed a $60 \%$ HFD, Spiro could not induce iBAT recruitment nor browning of WAT, probably due to the increase in the composition and fat content of the diet. Importantly, in this experimental setting, Fine was still able to affect iBAT mass. Compared to Spiro, Fine is characterized by a lower lipophilicity and higher polarity, which might lead to a different distribution in iBAT (adipose depot rich in mitochondria and water), potentially explaining a higher responsiveness of iBAT to Fine exposure. Furthermore, Fine is known to recruit different cofactors to the MR [6]. Recruitment of iBAT includes secretion of several batokines, which are known to promote adipogenesis, angiogenesis and immune cell interaction, but the influence of Fine on batokine expression is unknown. This observation may have relevant therapeutic implications in humans: in clinical practice, the use of Spiro is limited due to the risk of hyperkalemia and gynecomastia [6]; therefore, the prolonged use of steroidal MRAs requires careful monitoring of renal function, especially in patients with obesity and type 2 diabetes (T2D). Importantly, the recent clinical outcome trial FIDELIO-DKD showed that treatment with Fine reduced the progression of chronic kidney disease (CKD) and cardiovascular events in patients with CKD and T2D [7,8], with lower incidence of hyperkalemia than that observed previously with the classical steroidal MRA Spiro [7, 8]. Our current study suggests that Fine, through its direct effects on BAT, may contribute to gain a better glycemic control and prevent cardiovascular and renal complications in obese diabetic patients. Clinical studies on the metabolic 
A

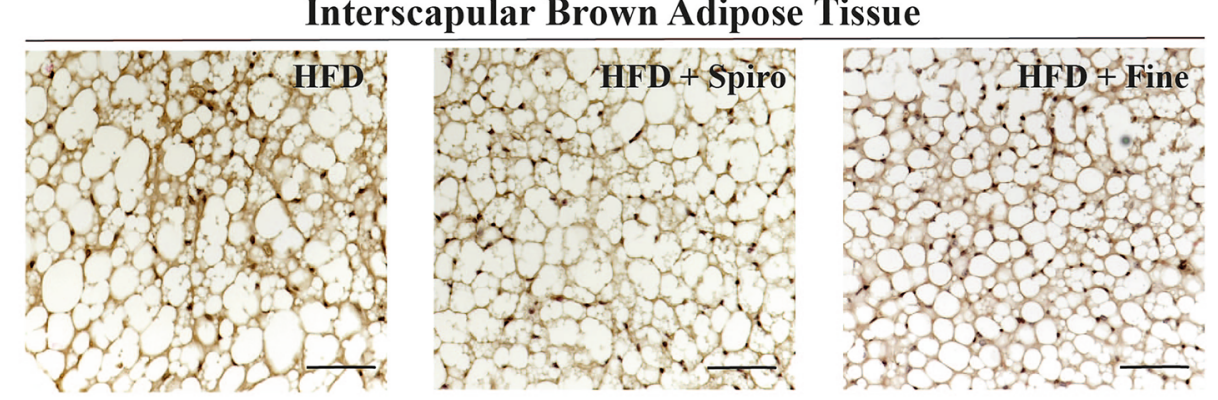

B

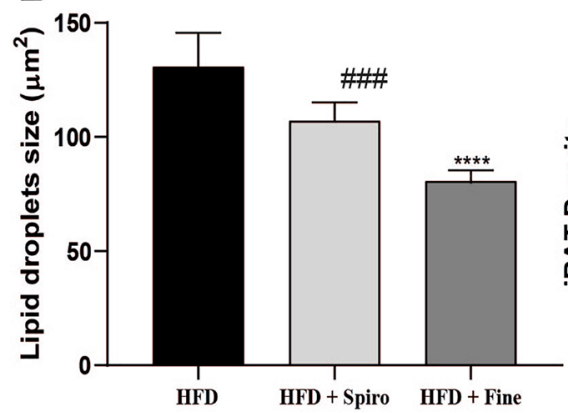

C

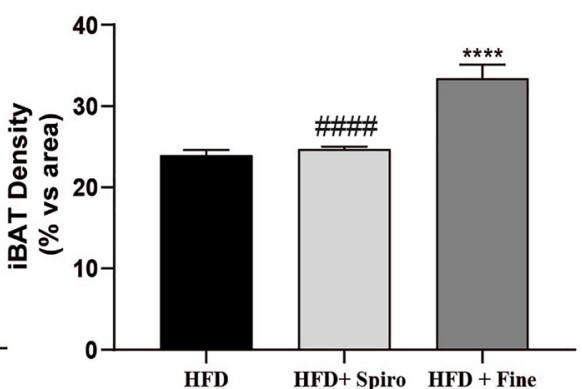

D

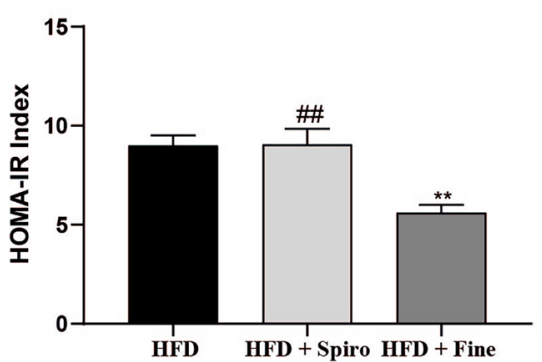

$\mathbf{E}$

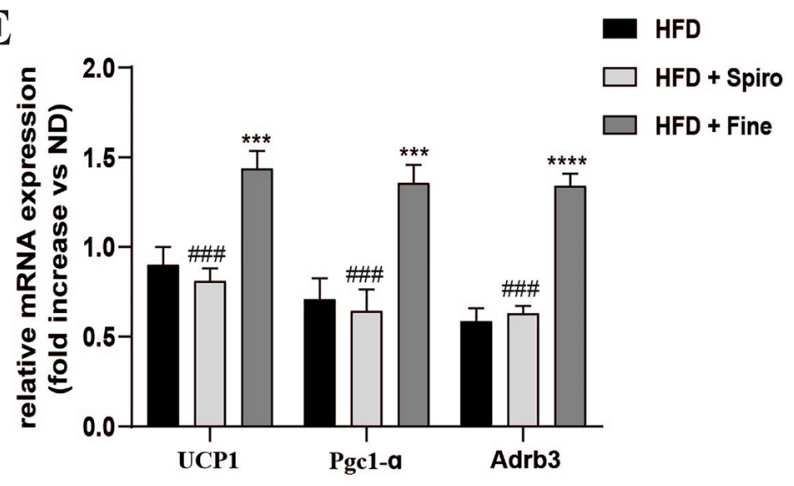

F

m HFD

$\square$ HFD + Spiro

$\square$ HFD + Fine

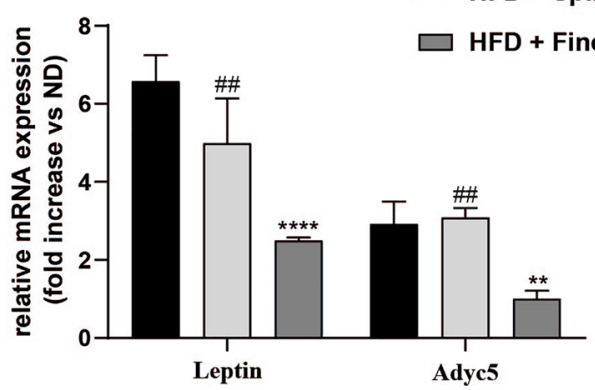

G
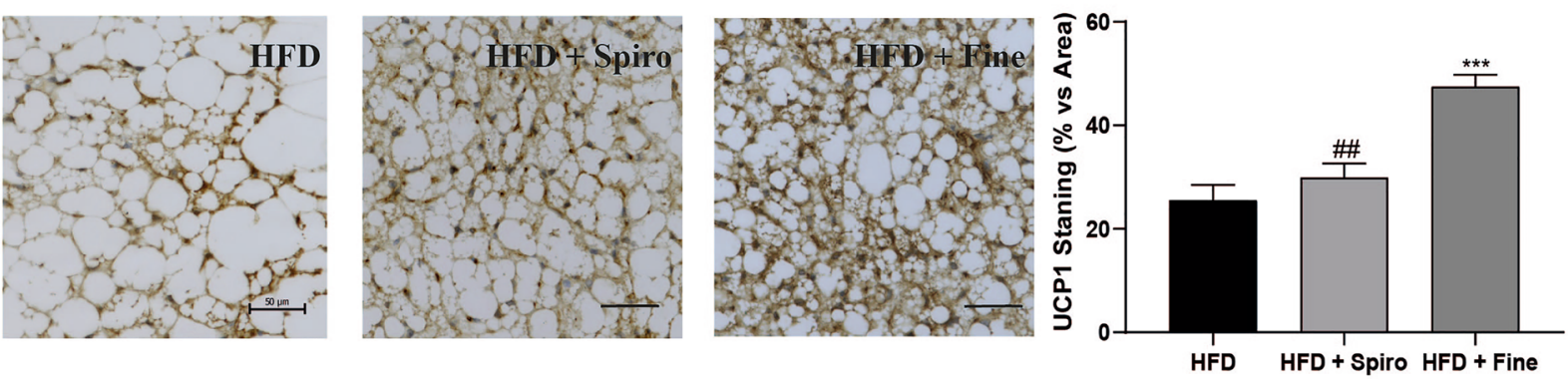

Fig. 1 Comparison between Spironolactone and Finerenone effects on iBAT depots of HFD-fed mice (A); H\&E staining of iBAT. Representative sections (scale bar $50 \mu \mathrm{m}$ ) from iBAT of all groups of mice $(n=6)(\mathbf{B})$; histological analysis of mean lipid droplets area $(n=6)(\mathbf{C})$; histological analysis of iBAT relative density $(n=6)$ (D); HOMA-IR index analysis (serum fasting glucose concentration $(\mathrm{mg} / 100 \mathrm{ml}) \times$ serum fasting insulin concentration $(\mathrm{mUI} / \mathrm{L})$ / $405(n=6)(\mathbf{E})$; qRT-PCR analysis of brown-fat-selective genes in
iBAT $(n=10)(\mathbf{F})$; qRT-PCR analysis of white-fat-selective genes in iBAT $(n=10)(\mathbf{G})$ UCP-1 immunostaining on iBAT. Representative sections from iBAT of all groups $(n=6)$. Positive area (UCP-1 staining) were selected and quantified by Image Pro Premier software. The quantification represents the average for six fields of each animal observed under 20-fold magnification. Scale bar $=50 \mu \mathrm{m} * * P<0.01$,

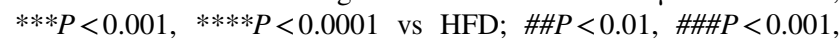
$\# \# \# P<0.0001$ vs HFD + Fine 


\section{A \\ INGUINAL FAT}
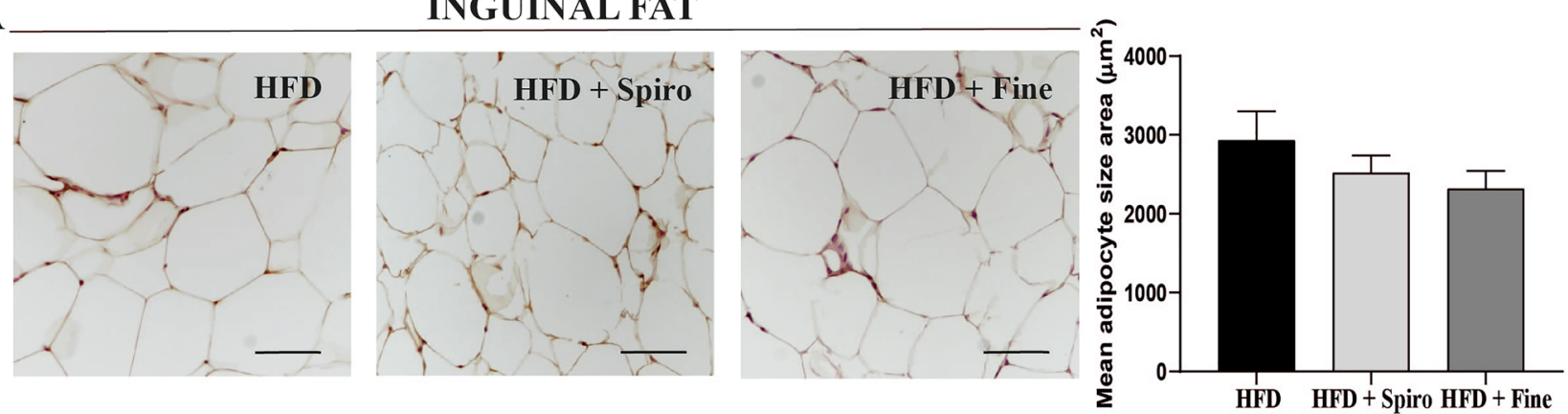

B

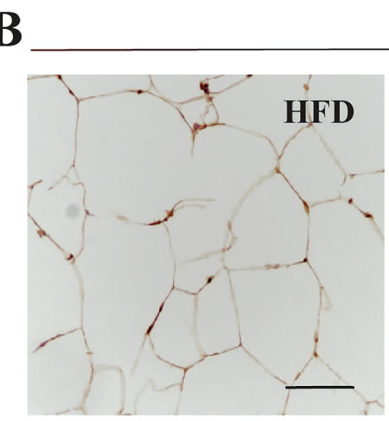

\section{GONADAL FAT}

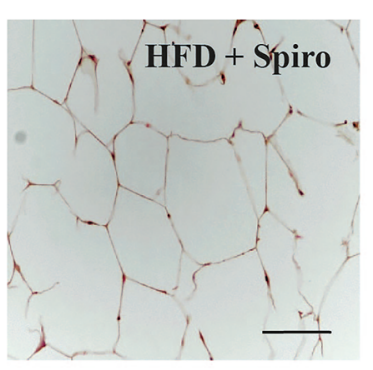

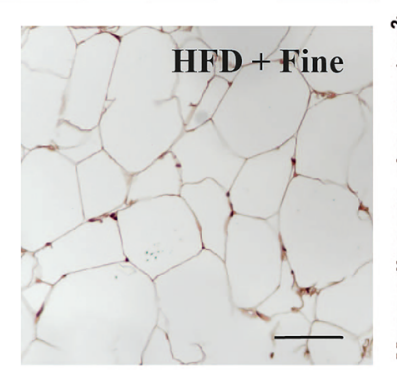

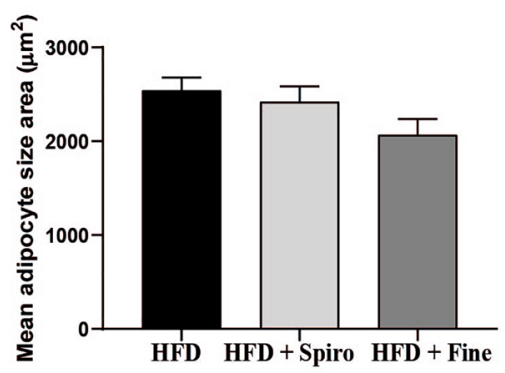

C

INGUINAL FAT

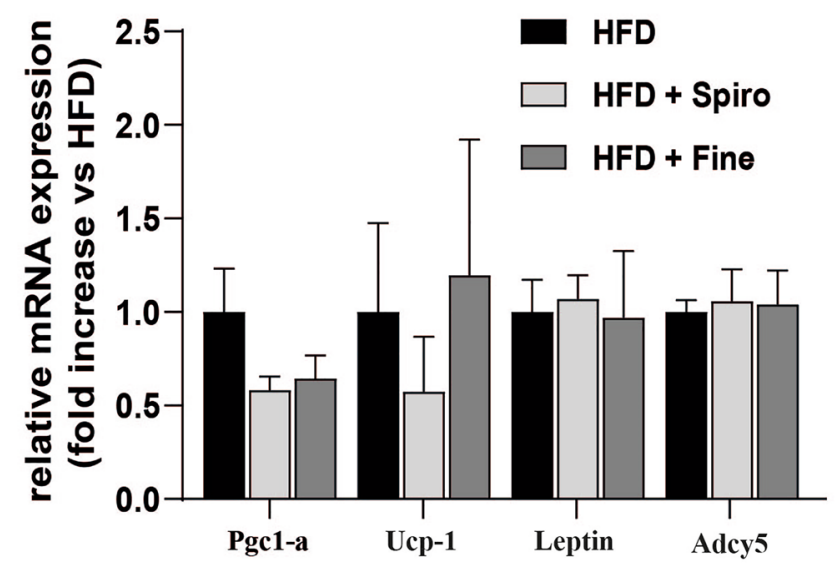

Fig. 2 Comparison between Spironolactone and Finerenone effects on WAT morphology of HFD-fed mice (A); H\&E staining on inguinal fat depots. Representative sections from inguinal fat depots of all groups of mice $(n=6$, scale bar $50 \mu \mathrm{m})$ and histological analysis of mean adipose cell size (B); H\&E staining on gonadal fat depots. Rep-

$\mathrm{C}$

outcomes of Fine, including BAT activity, in patients with obesity and/or T2D are deemed necessary to draw solid conclusions on this promising issue. Nevertheless, its safety profile, along with the encouraging preclinical

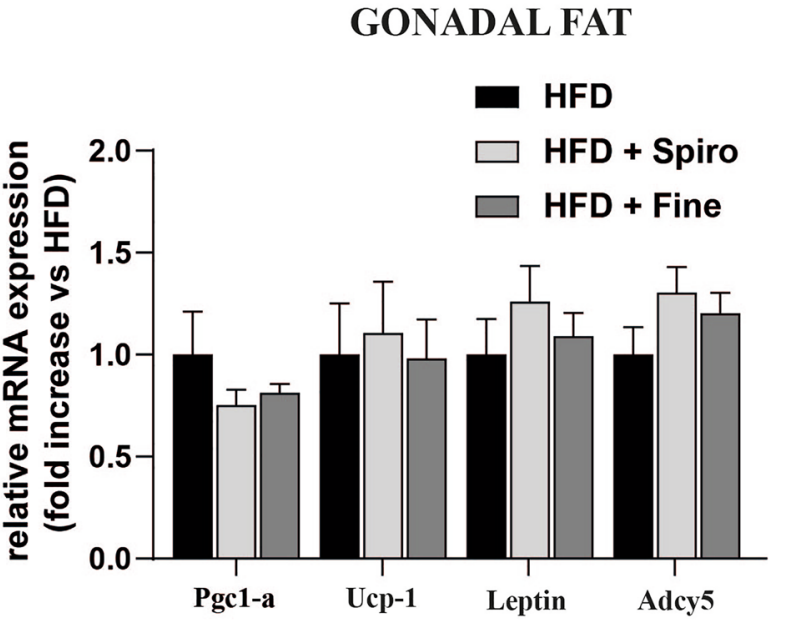

resentative sections from gonadal fat depots of all groups $(n=6$, scale bar $50 \mu \mathrm{m})$ and histological analysis of mean adipose cell size (C); qRT-PCR analysis of white fat- and brown fat-specific genes in inguinal and gonadal fat depots $(n=10)$

data presented in this paper, might qualify Fine for the treatment of human metabolic diseases associated with adipose tissue dysfunctions, potentially favouring cardiometabolic rehabilitation in this context. 
Acknowledgements This study was supported by the Italian Ministry of Health (Ricerca Corrente), and by MIUR (Progetti di Ricerca di Interesse Nazionale 2017- project code 2017A5TXC3 - to MC, Work Package Leader). We would like to thank Caterina Mammi and Stefania Gorini for technical support.

Author contributions V.M., A.F. and A.A. performed the in vitro and in vivo experiments. V.M., A.F., A.A. and M.C. conceived and designed the study. V.M., A.F., A.A. and M.C. performed data analysis and wrote the manuscript. P.K. contributed to drafting and critical review of the manuscript.

\section{Declarations}

Conflict of interest P.K. is a full-time employee of Bayer AG. M.C. received research grants from Bayer AG. The other authors have no conflict of interest.

Research involving human participants and/or animals Animal procedures were approved by the Italian National Institutes of Health Care and Use Committees (approval number 493/2016-PR). In the present study we did not perform research involving human participants.

Informed consent For this type of study, formal consent is not required.

\section{References}

1. Feraco A, Marzolla V, Scuteri A, Armani A, Caprio M (2020) Mineralocorticoid receptors in metabolic syndrome: From physiology to disease. Trends Endocrinol Metab 31:205-217. https:// doi.org/10.1016/j.tem.2019.11.006

2. Armani A, Cinti F, Marzolla V, Morgan J, Cranston GA, Antelmi A, Carpinelli G, Canese R, Pagotto U, Quarta C, Malorni W, Matarrese P, Marconi M, Fabbri A, Rosano G, Cinti S, Morag JY, Caprio M (2014) Mineralocorticoid receptor antagonism induces browning of white adipose tissue through impairment of autophagy and prevents adipocyte dysfunction in high-fat-dietfed mice. FASEB J 28:3745-3757. https://doi.org/10.1096/fj. $13-245415$

3. Marzolla V, Feraco A, Gorini S, Mammi C, Marrese C, Mularoni V, Boitani C, Lombes M, Kolkhof P, Ciriolo MR, Armani A, Caprio M (2020) The novel non-steroidal mr antagonist finerenone improves metabolic parameters in high-fat diet-fed mice and activates brown adipose tissue via ampk-atgl pathway. FASEB J 34:12450-12465. https://doi.org/10.1096/fj.202000164R

4. Fraulob JC, Ogg-Diamantino R, Fernandes-Santos C, Aguila MB, Mandarim-de-Lacerda CA (2010) A mouse model of metabolic syndrome: insulin resistance, fatty liver and non-alcoholic fatty pancreas disease (NAFPD) in C57BL/6 Mice Fed a High Fat Diet. J Clin Biochem Nutr 46:212-223. https://doi.org/10.3164/jcbn. 09-83

5. Thuzar M, Law WP, Dimeski G, Stowasser M, Ho KKY (2019) Mineralocorticoid antagonism enhances brown adipose tissue function in humans: A randomized placebo-controlled cross-over study. Diabetes Obes Metab 21:509-516. https://doi.org/10.1111/ dom. 13539

6. Agarwal R, Kolkhof P, Bakris G, Bauersachs J, Haller H, Wada T, Zannad F (2021) Steroidal and non-steroidal mineralocorticoid receptor antagonists in cardiorenal medicine. Eur Heart $\mathrm{J}$ 42:152-161. https://doi.org/10.1093/eurheartj/ehaa736

7. Bakris GL, Agarwal R, Anker SD, Pitt B, Ruilope LM, Rossing P, Kolkhof P, Nowack C, Schloemer P, Joseph A et al (2020) Effect of finerenone on chronic kidney disease outcomes in Type 2 diabetes. N Engl J Med 383:2219-2229. https://doi.org/10.1056/ NEJMoa2025845

8. Filippatos G, Anker SD, Agarwal R, Pitt B, Ruilope LM, Rossing P, Kolkhof P, Schloemer P, Tornus I, Joseph A et al (2021) Finerenone and cardiovascular outcomes in patients with chronic kidney disease and Type 2 diabetes. Circulation 143:540-552. https://doi.org/10.1161/CIRCULATIONAHA.120.051898

Publisher's Note Springer Nature remains neutral with regard to jurisdictional claims in published maps and institutional affiliations. 